\title{
Analysis on the Impact of R\&D Investment on Regional Innovation Achievements in the Yangtze River Delta
}

\author{
Chou $\mathrm{Mo}^{1, *}$ Chengyi $\mathrm{Pu}^{1}$ \\ ${ }^{1}$ School of Insurance, Central University of Finance and Economics \\ "Corresponding author.Email: pucy@cufe.edu.cn
}

\begin{abstract}
Technological innovation is the only way to maintain sustainable and high quality development in China. In order to further implement the policy of deepening scientific and technological reform, China has promulgated various policies to support innovation to promote the transformation and upgrading of the economic system. However, the effective conversion rate of Chinese enterprise patents is not high, which seriously affects the role of "catalyst" of national policy funds.Most of the existing research is focused on the analysis of inputs and outputs in the national market, But regional policies are not only typical, It is also more suitable for the reference of decision makers in the region. Based on data samples of Wuxi A stock market listed companies from 2010 to 2018, Using a fixed effect panel model, A comprehensive survey of the impact of $R \& D$ investment on enterprise innovation results, When the number of patent applications is found to be the explanatory variable, Government subsidies, net R \& D investment, profitability and asset size can significantly enhance innovation capabilities, But when the patent license number is the explanatory variable, Government subsidies cannot have a significant impact on the innovation results of enterprises. Revealed that many enterprises with subsidies to do "face project" to cope with the inspection phenomenon, this led to a significant reduction in the role of government subsidies. Therefore, This article suggests:(1) Wuxi government should adopt policy guidance, Converts the R\&D input mode to an enterprise-based input, (a) The mode of subsidization by the Government; (2) After the Government has released funds, To strengthen the supervision and management of funds, Promote enterprises to really invest this money in technological innovation; (3) The government should lead a diversified $\mathrm{R} \& \mathrm{D}$ investment system, Broaden the financing channels of small and medium-sized enterprises in the market.
\end{abstract}

Keywords: R\&D input, Government Subsidy, Innovation achievement, Influence mechanism, Yangtze River Delta

\section{长江三角洲 R\&D 投入对区域创新成果的影响分析}

\author{
莫愁 ${ }^{1, *}$ 蒲成毅 ${ }^{1}$
}

\author{
1 中央财经大学保险学院 \\ *通讯作者. 电子邮箱: Email: pucy@cufe.edu.cn
}

\section{摘要}

技术创新是我国保持可持续高质量发展的必经之路。为了进一步贯彻深化科技改革政策，我国先后颁布了各项 对创新的支持政策来推动经济体系转型升级，尽管国家财政科技拨款逐年递增，但是我国企业专利的有效转化 率并不高, 严重影响了国家政策资金“催化剂”的作用。现有的研究大多是针对全国市场对投入和产出之间进行 分析，然而区域性政策不仅具有典型性，也更适于区域内决策者参考。本文基于 2010 至 2018 年无锡 A 股市 场上市公司数据样本, 使用固定效应面板模型, 全面考察了 $\mathrm{R} \& \mathrm{D}$ 投入对企业创新成果的影响, 发现以专利申 请数为被解释变量时，政府补贴、净研发投入、盈利能力和资产规模都能显著地提升企业的创新能力，但以专 
利授权数为被解释变量时，政府补贴无法对企业的创新成果产生显著的影响。揭示了不少企业拿补贴款做着 “面子工程”应付检查的现象，这使得政府补贴的作用大大削减。因此，本文建议：（1）无锡政府应通过政策的 引导，将 R\&D 投入模式转换成一种以企业投入为主，政府补贴为辅的模式；(2) 政府在发放资金后，要加强 对资金的监督和管理，促使企业真正将这笔资金投入到技术创新中去;（3）政府应主导一个多元化的科技研发 投入体系，拓宽市场上中小微企业的融资渠道。

\section{关键字: $R \& D$ 投入, 政府补贴,创新成果, 影响机理, 长江三角洲}

\section{1 引言}

技术创新是我国保持可持续高质量发展的必经 之路。为了进一步贯彻深化科技改革政策, 强化以企 业为核心的科技创新体系, 我国先后颁布了各项对创 新的支持政策来扶持自主创新企业的发展, 推动经济 体系转型升级。在各项政策之中, 财政激励一直以来 是最为核心且有效的政策。据中国科技统计年鉴数据 显示，我国国家财政科技拨款从 2010 年的 4196.1 亿 元涨到了 2018 年的 9518.2 亿元, 年均增速达 $15 \%$ 。 尽管国家财政科技拨款逐年递增，但是我国企业方面 对于科技创新的支出却并不多, 专利的有效转化率也 不高, 大多数的专利都倒在了申请阶段, 2018 年中国 专利授权数仅占申请数的 $56 \%$, 且大部分聚集于长三 角和珠三角地区。

由于现在国内市场还不够完善, 经常出现失灵状 况，导致“搭便车”的现象频频发生，创新企业的科技 成果得不到保护, 最终创新的主体所获得的利益远远 小于市场总体, 甚至小于那些“搭便车”的企业, 最终 导致创新企业的研发动力不足。其次, 创新的风险大, 周期长, 导致市场内资金不敢进入，融资成本高，面 临严重的融资约束。

而政府的补贴能够起到很好的外部修正性作用, 这也是国际上比较通用的降低研发主体创新风险的 做法。 2018 年国家一共有 8326.7 亿元的科学技术支 出, 但是如此巨额的支出, 究竟对于企业的创新能力 有多大的提升, 作为创新主体的企业是否将这笔资金 投入了创新活动, 一直是学术界富有争议的话题, 至 今尚未得出统一结论。因此, 有必要对 R\&D 投入和 企业创新能力之间的关系进行进一步的研究。

\section{2 文献综述}

部分研究人员对政府的补贴持有积极的看法, 认 为政府补贴在一定程度上能够支持企业进行研发活 动 (Gonzalez, 2008), 同时降低企业的研发风险, 综 合来看能够大大降低企业的融资约束, 促进企业研发
成果的产生 (Lee, 2010)。另外, 基于信号理论视角, 发现政府补贴能够为企业释放有利信号, 从而帮助企 业更加容易获取外部的创新资源（夏清华和何丹, 2020)。

也有学者通过分析发现政府补贴仅仅在第一年 对被补贴企业的创新成果有正向的影响, 之后的补贴 对企业的创新能力没有显著的提升效果（Bergstrom, 2000 )。对全社会层面的企业而言，政府的补贴是具 有挤出效应的，因为对没有获得政府补贴的企业而言， 政府补贴大大增加了他们的研发风险，进而导致他们 退出或者减少研发活动（Tommy，2009）。

除了以上两种观点, 还有学者认为政府的补贴与 企业创新绩效存在 “倒 U 型” 的关系, 政府补贴金额 占营业收入的 $10 \%$ 20\%之间时存在激励效应的最优 值，当这个比例超过 $20 \%$ 时会存在政府补贴替代企业 自身投入研发资金的现象（Gullec，2003）。政府补贴 对企业创新效率的影响存在明显的双重门槛效应,且 当政府补贴力度介于 $0 \sim 0.0005$ 之间时,补贴效果最 优（任跃文，2019)。

最后，部分的学者在进行实证之后发现政府补贴 和创新绩效之间并没有显著的关系, 针对企业研发能 力的政府补贴并没有流入企业的研发资金中去, 大部 分的资金流向了企业的偿债能力上去, 导致最终的政 府补贴并没有提高企业的创新能力（Tzelepis \& Skuras，2004)。

\section{3 理论模型构建}

大多数的经验研究证实, 知识生产函数 (KPF) 是研究知识产出、区域性创新及其决定性因素的一个 富有说服力的经验模型。古典的知识生产函数实际上 就是一个标准的柯布-道格拉斯生产函数, 经过改进 和完善后成为了在投入产出法基础上研究知识产出 和区域创新的强有力的理论模型。该模型指出知识生 产主要投入要素为研发经费和研发人力资本, 并在综 合技术水平下转化为有价值的新知识和新技术。本文 
的理论模型就是基于修正的 Griliches-Jaffe 知识生产 函数, 模型具体表现形式如下:

$$
P I_{i}=A_{i} R D_{i}^{\alpha} H K_{i}^{\beta} e_{i}
$$

如果将 (1) 中修正的知识生产函数取对数, 则可 得到线性模型:

$$
\ln P I_{i}=a+\alpha \ln R D_{i}+\beta \ln H K_{i}+\mu
$$

其中 PI 为区域创新成果产出; $a$ 为综合技术水 平; $\mathrm{RD}$ 为区域创新投入的研发经费; $\mathrm{HK}$ 为区域创 新投入的人力资本; $i$ 代表样本观测单位; $\alpha$ 和 $\beta$ 代 表区域创新的研发经费投入和人力资本投入的产出 弹性; $\mu$ 代表随机扰动项。

实际上, 区域创新成果的产出除了和研发投入相 关外, 还取决于企业综合能力和社会环境的影响因素, 用 $\mathrm{Z}$ 来统一表示这些变量的影响。同时, 研发经费投 入可以细分为政府补贴中用来投入研发的部分和企 业净研发投入(ninvest), 而政府补贴又可以分为研发 补贴(rsub)和非研发补贴(nsub), 则式 (2) 可以表示为

$$
\begin{gathered}
\ln P I_{i}=a+\alpha_{1} \ln r s u b_{i}+\alpha_{2} \ln n s u b_{i} \\
+\alpha_{3} \ln \text { ninvest }_{i}+\beta \ln H K_{i}+\gamma Z_{i}+\mu
\end{gathered}
$$

由于一阶偏导 $\frac{\partial \ln P I}{\partial r s u b}, \frac{\partial \ln P I}{\partial n s u b}, \frac{\partial \ln P I}{\partial n i n v e s t}$ 均大于 等于零, 二阶偏导 $\frac{\partial^{2} \ln P I}{\partial r s u b^{2}}, \frac{\partial^{2} \ln P I}{\partial n s u b^{2}}, \frac{\partial^{2} \ln P I}{\partial n i n v e s t^{2}}$ 均小 于等于零, 因此 PI 是分别关于 rsub、nsub、ninvest 的 递增的凹函数, 根据此性质可以初步判断研发补贴、 非研发补贴和企业净研发投入都对企业创新成果产 出有正向促进作用。

\section{4 研究假设}

政府的补贴不仅能够起到直接缓解融资约束的 问题, 还能够间接给予市场投资者信心, 一定程度上 降低了信息不对称性。因此, 政府的补贴不论对于企 业本身还是对于外部的投资者而言都非常的重要, 它 不仅能增加企业的长期投资, 还能解决企业的融资约 束问题。因此, 我们据此猜测, 政府补贴能够一定程 度上缓解市场上的信息不对称性, 从而带来企业的创 新成果增加。因此提出假设:

$\mathrm{H} 1$ ：政府总补贴和企业创新能力呈正相关性

但是政府在发放补贴的时候同样也会出现各种
各样的信息不对称性, 且大多数的非研发补贴都是一 次性发放, 不具有任何的约束, 因此并不能提高企业 的创新研发能力。由于基于模型非研发补贴应能促进 企业创新产出，因此提出假设：

$\mathrm{H} 2$ ：政府研发补贴提高有助于提高企业创新能力

$\mathrm{H} 3 \mathrm{a}$ ：政府非研发补贴和企业创新能力无明显的关系

$\mathrm{H} 3 \mathrm{~b}$ ：政府非研发补贴有助于提高企业创新能力

扣除政府补贴之后的净研发投入可以视为企业 自身对于研发的重视程度, 而专利的产出可以一定程 度上代表企业的创新能力的高低。盈利能力和资产规 模则更加综合, 能够很直观的展现给外部其优秀的综 合能力。他不仅能代表企业能够有更多的资金投入研 发活动，也能够给外部投资者投资的信心，从而促进 企业的创新能力。因此提出假设:

$\mathrm{H} 4$ : 企业净研发投入增加有助于提高企业创新能力

$\mathrm{H} 5$ ：盈利能力提高有助于提高企业创新能力

H6: 资产规模量增加有助于提高企业创新能力

\section{5 实证分析}

\section{1 样本选择}

考虑 2010 年以前上市公司的创新成果产出值综 合较低, 2010 年以后专利等知识产权申请数和授权 数大幅提升, 所以选取无锡 2010-2018 年的 A 股上市 公司作为原始研究样本, 文中数据来自于 CSMAR 数 据库和公司年报数据, 缺失值通过国家知识产权局专 利库等来补齐, 使用 Stata 15.0 进行数据处理。

为了保证数据质量, 借鉴已有研究成果, 对原始 样本进行篮选, 最终保留了 53 家企业, 得到 401 个 样本点。本文最终保留 4812 条观测值, 为了消除极 端值对回归结果的影响, 本文对所有连续性变量进行 了上下双侧 $1 \%$ 的 Winsorize 处理。

\section{2 模型设定}

为检验前文提出的 5 个假设, 根据现有经验, 滞 后 1 期是补贴和企业研发经费投入对专利获得数的 最优滞后期, 因此, 本文设立因变量和公司规模为滞 后一期变量, 建立如下模型:

$$
\begin{gathered}
\ln \text { gpatent }_{t}=\beta_{0}+\beta_{1} \ln \text { rsub }_{t-1}+\beta_{2} \text { nsub }_{t-1} \\
+\ln \text { ninvest }_{t-1}+\ln \text { nroce }_{t-1}+\ln \text { asset }_{t-1} \\
+ \text { rgrate }_{t}+\text { girate }_{t}+\text { mtime }_{t}+\varepsilon_{t}
\end{gathered}
$$




\section{3 实证结果与分析}

\subsection{1 描述性统计}

本文主要的原始数据中公司年平均专利授权数 达到了 18.448 个, 最高的甚至达到了 224 个, 说明 无锡 A 股上市的企业具有较强的创新能力。20092018 年间平均每个公司获得了 1880 万的政府总补贴, 最高的达到了 4 亿元的补贴额, 说明无锡市政府很关 注本市的上市公司。研发补贴的平均值达到了 877 万, 占政府总补贴的 $47 \%$, 表明无锡的财政补贴较为有针 对性, 大部分的补贴额都是针对研发创新。企业净研 发投入值达到了 1.07 亿元, 表示无锡的上市企业本 身对于研发投入较大, 具有很强的创新研发意愿, 也 展现出了无锡市上市公司蓬勃的生命力。

\subsection{2 相关性分析与共线性检验}

经过对数化与缩尾处理后, 最终数据并未出现异 常值，除了非研发补贴和净研发投入在 5\%的水平下 与创新成果正相关, 其余自变量都是在 $1 \%$ 水平下和 创新成果正相关, 初步验证了假设 $\mathrm{H} 1 、 \mathrm{H} 2 、 \mathrm{H} 4$ 和 表 1 不同 $\mathrm{R} \& \mathrm{D}$ 投入对专利授权数的影响

\begin{tabular}{|c|c|c|c|}
\hline VARIABLES & lngpatent_w & VARIABLES & lngpatent_w \\
\hline - & - & L.lngsub_w & 0.1084 \\
\hline- & - & & $(1.5426)$ \\
\hline \multirow[t]{2}{*}{ L.lnnsub_w } & 0.0124 & - & - \\
\hline & 0.3951 & - & - \\
\hline \multirow[t]{2}{*}{ L.Inrsub_w } & 0.0501 & - & - \\
\hline & 1.196 & - & - \\
\hline \multirow[t]{2}{*}{ L.Inninvest_w } & $0.1405^{* *}$ & L.lnninvest_w & $0.2500 * * *$ \\
\hline & 2.3694 & & 4.1802 \\
\hline \multirow[t]{2}{*}{ L.Innroce_w } & $0.1961 * * *$ & L.Innroce_w & $0.1974 * * *$ \\
\hline & 3.3027 & & 3.4649 \\
\hline \multirow[t]{2}{*}{ L.Inasset_w } & $0.4693 * * *$ & L.lnasset_w & $0.4908 * * *$ \\
\hline & 3.9633 & & 4.182 \\
\hline \multirow[t]{2}{*}{ rgrate_w } & 0.3944 & rgrate_w & 0.5165 \\
\hline & 0.3566 & & 0.4839 \\
\hline \multirow[t]{2}{*}{ girate_w } & -0.1096 & girate_w & -0.3768 \\
\hline & $(-0.1084)$ & & $(-0.3885)$ \\
\hline \multirow[t]{2}{*}{ mtime_w } & $0.0539 * *$ & mtime_w & -0.1927 \\
\hline & 2.1231 & & $(-1.5827)$ \\
\hline \multirow[t]{2}{*}{ Constant } & $-12.1258 * * *$ & Constant & $-10.1290 * * *$ \\
\hline & $(-5.8007)$ & & $(-3.4634)$ \\
\hline
\end{tabular}

Z-statistics in parentheses

$* * * \mathrm{p}<0.01, * * \mathrm{p}<0.05, * \mathrm{p}<0.1$
H5，初步说明总补贴、研发补贴、净研发投入、盈利 能力是和企业创新能力是成正相关关系的。

且通过共线性检验, 各变量的方差膨胀因子 VIF 均值为 2.01 , 最大值为 2.66 , 均未超过 10 , 表明各变 量间不存在严重的多重共线性，进一步证明了模型的 合理性。

\subsection{3 回归结果分析}

对样本通过 $\mathrm{F}$ 检验、LM 检验和 Hausman 检验 后，本文采取固定效应模型进行回归。在 stata 中进 行固定效应面板回归之后, 得到表 1 中的回归分析结 果。表 1 展示了不同类型的 R\&D 投入对企业创新成 果的影响。

首先观察含有政府总补贴的回归结果，虽然政府 总补贴的影响是正向的, 而且系数较大, 但是此变量 的显著性检验并未通过。在方程整体显著性检验通过 的情况下, 滞后一期的净研发投入、企业盈利能力和 资产规模都在 $1 \%$ 水平下显著，且系数为正。资产规 模的影响系数最大, 达到了 0.49 , 其次是净研发投入, 最后是盈利能力。对比将政府总补贴分为研发补贴和 非研发补贴的回归结果，研发补贴和非研发补贴的系 数都为正, 但是显著性检验结果均未通过, 而滞后一 期的净研发投入、企业盈利能力和资产规模仍然在 $1 \%$ 水平下显著, 资产规模的系数仍然是最大, 盈利能力 的系数几乎不变, 净研发投入的影响系数稍有减少, 但是仍维持在较高的系数水平。

综合来看，这两个面板回归结果支持了假设 H4 H5、H6，即企业净研发投资的增加，企业盈利能力的 提高以及企业资产规模量增加有助于提高企业创新 能力，而并没有有效的证据支持假设 $\mathrm{H} 1 、 \mathrm{H} 2 、 \mathrm{H} 3$ 。 可能原因是政府在发放补贴的时候会出现各种各样 的信息不对称性，企业在申请补贴的时候有可能虚报 资金甚至编造课题，研发的补贴的发放和验收行为不 严谨，对于企业的约束作用不强而导致的。而非研发 补贴由于其不具有约束性, 且大多数的非研发补贴都 是一次性发放, 因此不仅留下了很多寻租的空间, 还 并不能提高企业的创新研发能力。

\subsection{4 稳健性检验}

为检验该结论的稳健性, 本文对被解释变量进行 了更换，将被解释变量由专利授权数量（gpatent）替 
表 2 具体变量的含义及计算方法

\begin{tabular}{|c|c|c|c|}
\hline 变量 & 名称 & 含义 & 具体计算方法 \\
\hline \multirow{5}{*}{ 因变量 } & Ingpatent & 创新成果 & $\begin{array}{c}\text { 当年独立和联合获得的发明专利+实用新型专利 } \\
+ \text { 外观设计数量, 取对数 }\end{array}$ \\
\hline \multirow{5}{*}{ 自变量 } & Ingsub & 政府补贴 & 当年总补贴, 取对数 \\
\cline { 2 - 5 } & Inrsub & 政府研发补贴 & 与研发相关的补贴之和, 取对数 \\
\cline { 2 - 5 } & Innsub & 政府非研发补贴 & 与研发无关的补贴之和, 取对数 \\
\cline { 2 - 5 } & Inninvest & 企业净研发投入 & 研发支出-政府补贴, 取对数 \\
\cline { 2 - 5 } & Innroce & 扣除非经常性损益后净投资收益率 & 扣非母公司净利润/期初期末净投入资本均值 \\
\hline \multirow{3}{*}{ 制变量 } & Inasset & 公司规模 & 总资产, 取对数 \\
\cline { 2 - 5 } & grate & 无锡市财政收入增长率 & 当年财政收入/上年财政收入-1 \\
\cline { 2 - 5 } & girate & 无锡市 GDP 增长率 & 当年 GDP/上年 GDP-1 \\
\cline { 2 - 5 } & mtime & 已上市时间 & 年份-企业上市年份 \\
\hline
\end{tabular}

换为专利申请数量 (fpatent)。与专利授权数相似, 同 样在使用专利申请数指标之前, 对样本进行对数化和 缩尾处理, 得到指标 Infpatent.

由于专利申请比专利获得更加容易, 2018 年中 国专利授权数仅占申请数的 $56 \%$, 因此以专利申请数 为被解释变量的回归结果一定效果更好。在含有政府 总补贴的回归结果中, 滞后一期的政府总补贴、企业 净研发支出、企业盈利能力、总资产规模都对企业的 创新研发能力有正向的促进作用，且在 $1 \%$ 的显著水 平下有较高的影响系数, 其影响效果最强的仍然是总 资产规模, 系数达到了 0.49 , 其次是企业净研发支出, 最后是企业的盈利能力。在将总补贴分为研发补贴和 非研发补贴的回归结果之中, 滞后一期的研发补贴、 非研发补贴、净研发投资、盈利能力和资产规模都对 企业的创新能力有显著的正向影响效果, 都在 $1 \%$ 的 水平下显著。值得注意的是, 对于专利申请数而言, 研发补贴相对于非研发补贴有更好的促进作用, 可能 原因是研发补贴的申领是分期的且需具有验收和跟 进等约束行为。

综合来看, 资产规模的影响效果最为显著, 盈利 能力和企业净研发投入的影响效果相近, 但都大于研 发补贴、非研发补贴以及总补贴的效果, 原因可能是 相比于外部的融资, 内部的融资更具有约束力, 在这 个市场失灵且信息不对称的情况下, 净研发投入更能 体现一个企业创新的意愿。外部的政府补贴对专利的 申请数有一定的影响力, 并不代表政府补贴的有效性, 反而更能体现企业的寻租以及做“面子工程”的情况 存在, 以专利授权数为被解释变量更具有说服力。

\section{6 结论与展望}

以专利授权数为被解释变量时, 无论是研发补贴、 非研发补贴还是政府的总补贴, 都无法对企业的创新 成果产生显著的影响，但是企业的净研发投入、盈利 能力和资产规模都有显著的正向影响, 按影响效果来 看，资产规模产生的效果最明显，企业净研发投入和 企业的盈利能力对创新能力的提升效果相近。以专利 申请数为被解释变量时, 总补贴、研发补贴、非研发 补贴、净研发投入、盈利能力和资产规模都能显著地 提升企业的创新能力。

这使我们能更理性的认识政府补贴的效果, 虽然 研发补贴是针对企业创新活动的补助, 但是由于市场 信息不对称的存在，不少企业仍然会选择拿着补贴款 做着“面子工程”应付检查，这使得研发补贴的作用大 大削减。虽然非研发补贴能够使得企业一定程度上缓 解融资约束, 带动企业研发, 但是从结果来看对创新 成果和盈利能力的提升是不显著的。市面上的政府补 贴中存在大量的类似于纳税补贴、环保补贴、拆迁补 贴等项目, 这些补贴没有将企业的成果与之绑定，缺 乏激励措施, 使得最终的产出效果并不理想。对比看 来，企业自身的资产规模、盈利能力和净研发投入更 能促进创新成果的产出, 这是因为只有与企业自身努 力和付出相绑定, 才能确保产出成果, 内部融资的约 束性使得企业研发部门寻租的可能性大大减小, 进而 导致同等的付出收获同等的成果。

基于以上结论，我们认为无锡政府在制定 $R \& D$ 补贴时，应鼓励企业增加 $\mathrm{R} \& \mathrm{D}$ 投入，可以采取有效 
措施，通过政策的引导，将 R\&D 投入模式转换成一 种以企业投入为主，政府补贴为辅的模式，如通过对 税收减免等间接工具的作用鼓励企业进行 R\&D 投入。 同时, 政府在发放资金后, 要加强对资金的监督和管 理，促使企业真正将这笔资金投入到技术创新中去， 更好的激励企业产出创新成果。此外, 政府应主导一 个多元化的科技研发投入体系, 拓宽市场上中小微企 业的融资渠道，使得总资产量小的企业也能够增强自 主研发能力, 形成政府带头, 引领社会资金和社会力 量共同参与 $\mathrm{R} \& \mathrm{D}$ 的投入中来, 更好的促进创新产出。

\section{致谢}

本文是北京高校高精尖学科 “战略经济与军民融 合” 交叉学科 (GJJ2019163)、中央财经大学 “一流学 科” 建设项目 “巨灾风险管理与自然灾害治理现代化 研究”，2020 年度国家民委民族研究项目 “后脱贫时 代西部藏区乡村振兴风险与内置金融研究（2020GMB-030)”的阶段性研究成果。

\section{参考文献}

[1] Xulia Gonzalez, Consuelo Pazo. Do public subsidies stimulate private $R \& D$ spending? [J]. Research Policy, 2008, 37(4): 371-389.

[2] Eui Young Lee, Beom Cheol Cin. The effect of risksharing government subsidy on corporate R\&D investment: Empirical evidence from Korea[J]. Technological Forecasting \& Social Change, 2010, 77(6): 881-890.

[3] 夏清华,何丹.政府研发补贴促进企业创新了吗一 一信号理论视角的解释 [J]. 科技进步与对 策,2020,37(01):92-101.

[4] Fredrik Bergström. Capital Subsidies and the Performance of Firms[J]. Small Business Economics, 2000, 14(3): 183-193.

[5] Tommy H.Clausen. Do subsidies have positive impacts on $R \& D$ and innovation activities at the firm level? [J]. Structural Change and Economic Dynamics, 2009, 20(4): 239-253.

[6] Guellec D., Potterie B. V. P. The Impact of Public $R \& D$ Expenditure on Business R\&D $[J]$. Economics of Innovation and New Technology. 2003, 12(3): 225-243.

[7] 任跃文.政府补贴有利于企业创新效率提升吗— 一基于门槛模型的实证检验 [J].科技进步与对 策,2019,36(24):18-26.

[8] Dimitris Tzelepis, Dimitris Skuras. The effect of regional capital subsidies on firm performance: an empirical study[J]. Journal of Small Business and Enterprise Development. 2004, 11(1): 121-129.

[9] Kenneth J. Arrow. Economic Welfare and the allocation of resources for innovation [M]. The Rate and Direction of Inventive Activity: Economic and Social Factors: A Conference of the Unive, 1962 , Vol., p.609-626.

[10] Mansfield, Edwin, Mark Schwartz, and Samuel Wagner. Imitation Costs and Patents: An Empirical Study[J]. The Economic Journal 1981, 91 (364): 907-918. 